\title{
The Application of Residue Number System to the Design of High-Speed FIR Filters
}

\author{
Zhe $\operatorname{Jin}^{1,}$, Jie $\mathrm{Wu}^{2}$ \\ ${ }^{1}$ School of Automation and Electrical Engineering, Zhejiang University of Science \\ and Technology, Hangzhou, 310023, China \\ ${ }^{2}$ Hangzhou Fuyang Vocational Education Center, Hangzhou, 311400, China \\ aemail: zjuz@163.com
}

Keywords: Finite Impulse Response Filter; Residue Number System; Digital Signal Processing; Chinese Remainder Theorem

\begin{abstract}
Finite impulse response (FIR) filters implemented in binary weighted number system suffer from the carry propagation delay. To accelerate the FIR filtering process, a design method is proposed on the basis of residue number system (RNS), which has been considered as an important methodology of high-speed computation. The arithmetic operations are decomposed into a set of small modular operations, which can be performed by several sub-filters in parallel. At the end of filtering, the residue signals are converted to conventional binary notation via Chinese remainder theorem. The performance of the presented RNS FIR filter is evaluated by computer simulation. The results show that frequency specifications are satisfied and the time delay of arithmetic operations is substantially shortened.
\end{abstract}

\section{Introduction}

Finite impulse response (FIR) filters are one of the most common building blocks in digital signal processing (DSP) applications. Sometimes FIR filters are preferable to infinite impulse response (IIR) filters due to their useful attributes such as stable structures. Usually, to meet certain frequency specifications, the order of FIR filters is relatively high. The high order results in a great number of multiplications and additions, which limit the speed of FIR filtering [1].

The residue number system (RNS) is non-weighted number system, which has been recognized as an efficient methodology of high-speed signal processing for its inherent parallelism. In RNS, a large number is represented by a set of small residues. The arithmetic operations on large numbers can be done by performing operations on these small residues in parallel [2][3]. Since the arithmetic operations in different modular channels are independent of each other, there is no carry propagation among them. Therefore, the arithmetic operations are substantially accelerated. RNS is good at addition, subtraction, and multiplication, while some other operations, such as division and magnitude comparison, are difficult within RNS. The superiority of RNS in the design of high-speed FIR filters relies on the fact that only addition and multiplication are involved in the filtering [4].

A method of designing high-speed FIR filters based on RNS is proposed in this study. Firstly, conventional binary signal is decomposed into residue signals. Then the residue signals are processed by sub-filters in different modular channels. Finally, the outputs of the sub-filters are converted to traditional signal via Chinese remainder theorem (CRT) [5]. The performance of the proposed scheme is validated by computer simulation. 


\section{Background of Residue Number System}

An RNS is characterized by $K$-tuple moduli set $P=\left\{p_{1}, p_{2}, \cdots, p_{K}\right\}$, in which $p_{i}$ 's $(i=1,2, \ldots$, $K)$ are co-prime positive integers. The dynamic range of RNS is defined as

$$
M=\prod_{i=1}^{K} p_{i} .
$$

Each integer $0 \leq X<M$ can be uniquely represented as a residue set $X \square\left\langle x_{1}, x_{2}, \ldots, x_{K}\right\rangle$, where $x_{i}$ is the residue of $X$ with respect to $p_{i}$, which is noted by $x_{i}=|X|_{p_{i}}$.

As the coefficients of an FIR filter may be positive or negative, it is required to represent positive numbers as well as negative numbers in RNS. If a signed integer $X$ satisfies one of two relations [6]

$$
\left\{\begin{array}{ll}
-\frac{M-1}{2} \leq X \leq \frac{M-1}{2}, & \text { if } M \text { is odd } \\
-\frac{M}{2} \leq X \leq \frac{M}{2}-1, & \text { if } M \text { is even }
\end{array},\right.
$$

then its residue $x_{i}$ is calculated as

$$
x_{i}=\left\{\begin{array}{ll}
|X|_{p_{i}}, & \text { if } X \geq 0 \\
p_{i}-|-X|_{p_{i}} & \text { if } X<0
\end{array}\right. \text {. }
$$

For two given operands $X, Y \in[0, M)$, their arithmetic modular operation is performed as

$$
|X \square Y|_{M} \square\left\langle\left|x_{1} \square y_{1}\right|_{p_{1}},\left|x_{2} \square y_{2}\right|_{p_{2}}, \ldots,\left|x_{K} \square y_{K}\right|_{p_{K}}\right\rangle
$$

where $\sqcup$ denotes addition, subtraction or multiplication. It is seen that the operation on large numbers can be done by several operations on smaller residues independently. Parallelism and small digit size explain why RNS is advantageous to the implementation of high-speed FIR filters.

\section{The Design of FIR Filters Using RNS}

An FIR filter is described by

$$
Y_{n}=\sum_{j=0}^{N} A_{j} X_{n-j}
$$

where $X_{n}$ is the input to the filter, $Y_{n}$ is the output, $N$ is the filter's order, and $A_{j}$ denotes the coefficients. The structure of the RNS FIR filter is shown in Fig.1. The first step is to translate the conventional binary signal $X$ into residue signals $\left\langle x_{1}, x_{2}, \ldots, x_{K}\right\rangle$. And then the residue signals are processed by $K$ sub-filters in parallel. Finally, the outputs of the sub-filters are combined to binary representation by reverse conversion.

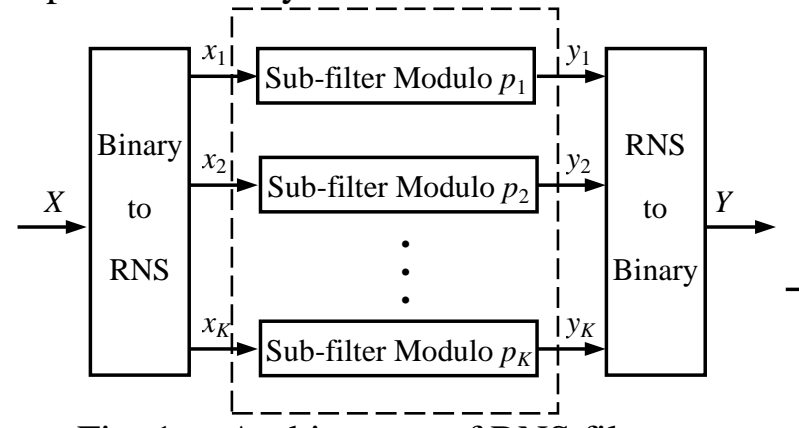

Fig. 1. Architecture of RNS filter

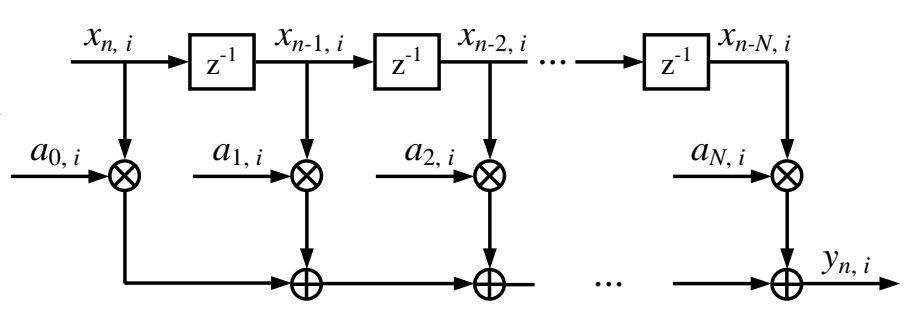

Fig. 2. Structure of modulo $p_{i}$ sub-filter

The sub-filter in modulo $p_{i}$ channel is described by 


$$
y_{n, i}=\left|\sum_{j=0}^{N} a_{j, i} x_{n-j, i}\right|_{p_{i}}
$$

where $a_{j, i}=\left|A_{j}\right|_{p_{i}}, \quad x_{n-j, i}=\left|X_{n-j}\right|_{p_{i}}, \quad y_{n, i}=\left|Y_{n}\right|_{p_{i}}$, and $i=1,2, \cdots, K$. Fig. 2 shows the structure of sub-filter modulo $p_{i}$, where $\oplus$ and $\otimes$ denote modular addition and modular multiplication respectively, and $z^{-1}$ stands for a unit delay. The building block of the sub-filter is a modular multiplicator followed by a modular adder [7].

Reverse conversion is the process of translating from residue representation to conventional binary notation. It is one of the difficult operations in RNS. The existing reverse conversion methods are classified into two categories. One is mixed radix conversion (MRC). The other is on the basis of CRT. The implementation of MRC is simpler, but the conversion speed is slower because of its serial mechanism. The operations of CRT are in parallel and higher conversion speed can be acquired [8]. Here we adopt CRT as the principle of the reverse conversion. The sub-filters' residue outputs $\left\langle y_{1}, y_{2}, \ldots, y_{K}\right\rangle$ is converted to the common binary signal $Y$ by

$$
Y=\left|\sum_{i=1}^{K} y_{i} t_{i} P_{i}\right|_{M}
$$

where $P_{i}=\frac{M}{p_{i}}$ and $t_{i}$ satisfies $\left|t_{i} P_{i}\right|_{p_{i}}=1$.

Moduli set selection is another issue that affects the performance of the DSP system. The form of moduli set directly impact on the complexity of arithmetic operations [9]. In our design, the moduli set $\left\{2^{m}-1,2^{m}, 2^{m}+1\right\}$ is adopted for its advantages in the implementation of modular operations. Addition modulo $2^{m}$ is just generic unsigned addition, and addition modulo $2^{m}-1$ is very similar to common 1s-complement addition. Addition modulo $2^{m}+1$ can be realized by the diminished-one representation.

\section{Performance Validation}

Let's take an example to illustrate the above scheme and then validate the performance of the filter by computer simulation. Consider 31st order bandpass FIR filter. The normalized passband frequency is $[0.3,0.6]$. Symmetrical coefficients are utilized to provide linear phase and simplify the design of arithmetic operators. A part of coefficients are listed in the second column of table 1. The moduli set is chosen as $\left\{2^{8}-1,2^{8}, 2^{8}+1\right\}$. So the dynamic range is

$$
M=\left(2^{8}-1\right) \times 2^{8} \times\left(2^{8}+1\right) \approx 2^{24}
$$

Eq. 8 signifies that the resolution of the FIR filter is about 24 bits. The bit width of the coefficients and the input signal are allocated as 11 and 8 respectively. The coefficients are quantized to integers between -1023 and 1023 . The integer and RNS representations of the coefficients are shown in table 1 . The process of input signal is similar.

Table 1 Filter coefficients

\begin{tabular}{cccc}
\hline & $\begin{array}{c}\text { Decimal } \\
\text { representation }\end{array}$ & $\begin{array}{c}\text { Integer } \\
\text { representation }\end{array}$ & $\begin{array}{c}\text { RNS representation } \\
<a_{j, 1}, a_{j, 2}, a_{i, 3}>\end{array}$ \\
\hline$A_{0}=A_{31}$ & -0.00277864786039 & 16776948 & $<243,244,245>$ \\
$A_{1}=A_{30}$ & -0.00016011315957 & 0 & $<0,0,0>$ \\
$\ldots$ & $\ldots$ & $\ldots$ & $\ldots$ \\
$A_{14}=A_{17}$ & -0.14025833424407 & 16776321 & $<126,129,132>$ \\
$A_{15}=A_{16}$ & 0.22432870996504 & 1023 & $<3,255,252>$ \\
\hline
\end{tabular}




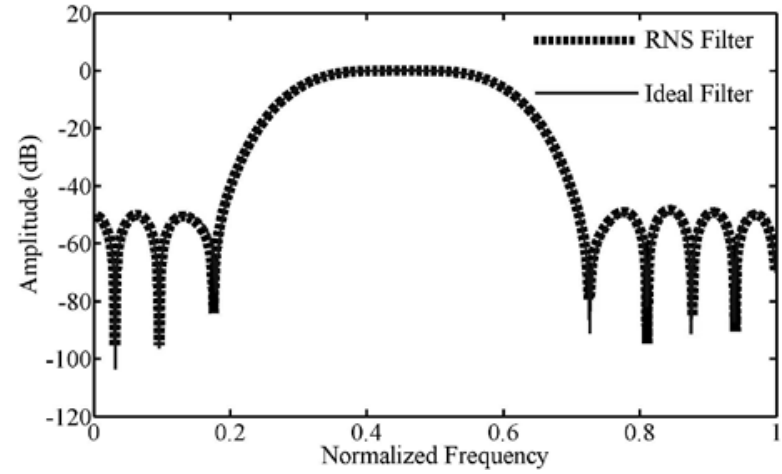

Fig. 3. Amplitude response

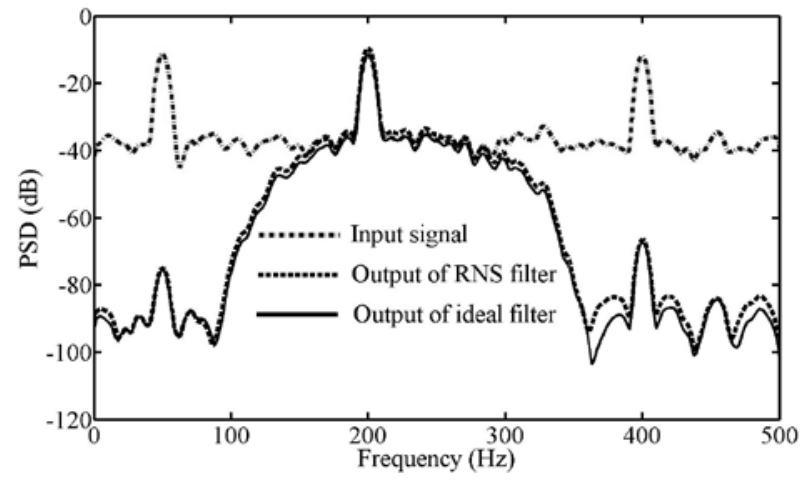

Fig. 4. PSD of input and output signal

The amplitude response of the RNS FIR filter is compared with the same order ideal filter in Fig. 3. Here the ideal filter means that the coefficients are represented by high precision decimal numbers. Fig. 3 shows that the amplitude response of the RNS filter is almost the same as that of the ideal filter. The bandpass filters are driven by the input signals which consist of $50 \mathrm{~Hz}, 200 \mathrm{~Hz}$, and $400 \mathrm{~Hz}$ sinusoidal waves. White Gaussian noise, whose signal-to-noise ratio is $10 \mathrm{~dB}$, is added to the input signals. The power spectral density (PSD) of input and output signals is exhibited in Fig. 4. It is demonstrated that the RNS filter effectively suppresses the signals out of the passband. However, the RNS filter's PSD performance is slightly inferior to the ideal filter due to its bit width limitation.

\section{Conclusion}

To implement high-speed FIR filters, a design method based on residue number system is proposed. The arithmetic operations in filtering are divided into a set of small operations, which are performed by several sub-filters in parallel. Therefore, the signal processing is accelerated substantially. The performance of the proposed scheme is validated by computer simulation.

\section{Acknowledgement}

This paper was supported by China Scholarship Council.

\section{References}

[1] W. Kenneth Jenkins and Benjamin J. Leon. The Use of Residue Number Systems in the Design of Finite Impulse Response Digital Filters [J]. IEEE Transactions on Circuits and Systems, 1977, 24(4): 191-201.

[2] Pedro Miguens Matutino, Ricardo Chaves, and Leonel Sousa. An Efficient Scalable RNS Architecture for Large Dynamic Ranges [J]. Journal of Signal Processing Systems, 2014, 77(3): 191-205.

[3] Jienan Chen and Jianhao Hu. Energy-Efficient Digital Signal Processing via Voltage-Overscaling-Based Residue Number System [J]. IEEE Transactions on Very Large Scale Integration (VLSI) Systems, 2013, 21(7), 1322-1332.

[4] Richard Conway and John Nelson. Improved RNS FIR Filter Architectures [J]. IEEE Transactions on Circuits and Systems-II, 2004, 51(1): 26-28.

[5] Shaoqiang Bi and Warren J. Gross. The Mixed-Radix Chinese Remainder Theorem and Its Applications to Residue Comparison [J]. IEEE Transactions on Computers, 2008, 57(12): 1624-1632.

[6] Amos Omondi and Benjamin Premkumar. Residue Number Systems: Theory and Implementation [M]. London: Imperial College Press, 2007. 
[7] Stanisław J. Piestrak and Piotr Patronik. Design of Fault-Secure Transposed FIR Filters Protected Using Residue Codes [A]. 17th Euromicro Conference on Digital System Design [C]. Verona, 2014: 27-29.

[8] Hector Pettenghi, Ricardo Chaves, and Leonel Sousa. RNS Reverse Converters for Moduli Sets with Dynamic Ranges up to (8n+1)-Bit [J]. IEEE Transactions on Circuits and Systems-I, 2013, (60)6: 1487-1500.

[9] Gavin Xiaoxu Yao, Junfeng Fan, Ray C.C. Cheung, and Ingrid Verbauwhede. Novel RNS Parameter Selection for Modular Multiplication [J]. IEEE Transactions on Computers, 2014, 63(8): 2099-2105. 